\title{
Enseñanza en la Era Digital: La Empatía Docente y el Aprendizaje Colaborativo
}

\author{
Teaching in the Digital Age: Teacher's empathy and Collaborative Learning
}

\author{
Verónica Paola Rossado Espinoza \\ Universidad Ricardo Palma, Perú \\ paola.rossado@gmail.com
}

\begin{abstract}
We are living in the technology and knowledge society, marked by networks, information and collaboration. As a result of the digital revolution, the learning is developing into social connective context with new activities among students. They have a capacity to interact, share and work together, for discuss, analysis and evaluate options. If this integration is monitoring by teacher and they receive special motivation, it could develop different skills. This paper discusses the idea that collaborative learning and teacher's empathy improve the significant learning.
\end{abstract}

Keywords: Empatía; Aprendizaje colaborativo; Enseñanza digital; Aprendizaje conectivo; Trabajo colaborativo

\section{Introducción}

La sociedad de la información, las tecnologías y las comunicaciones, influyen en diversas actividades de la vida diaria; en el ámbito educativo, ofrecen gran gama de posibilidades tanto para profesores como estudiantes. Los tradicionales métodos de aprendizaje se han visto fortalecidos ante la incorporación de nuevas herramientas digitales, donde las aulas tienen la oportunidad de convertirse en espacios amigables de participación y debate, potencialmente atractivos a los estudiantes.

En la actualidad, las comunicaciones y el internet brindan un recurso casi infinito, los usuarios interactúan y obtienen una respuesta inmediata en cualquier lugar que se encuentren. En este entorno, desarrollan nuevas aptitudes y destrezas; si bien es cierto que a veces las redes sociales y los juegos electrónicos generan un efecto negativo y de distracción entre los jóvenes, será labor de los educadores, orientar el uso de estas las herramientas en forma responsable.

Los diversos medios de comunicación y el internet, actualmente son utilizados como recursos en la educación, y pueden fortalecer el aprendizaje significativo. Valiéndose de sistemas de colaboración e intercambio de información, los estudiantes tienen la libertad de interactuar y desarrollar todo su potencial creativo.

El presente trabajo tiene por objetivo establecer estrategias de enseñanza que utilicen tecnologías y comunicaciones, con el propósito de mejorar el aprendizaje a través del debate, la participación y la colaboración en el aula. Transformar la figura del profesor transmisor de saberes, para convertirlo en un promotor de ideas creativas e innovadoras, resaltando características de empatía y actividades de cooperación en el aula.
Es importante que el profesor reconozca las habilidades de cada alumno, y mantenga un ambiente de cordialidad y confianza. Chiarella afirma: "Así el aula se constituye en un campo de interacción de ideas, representaciones y valores en donde los alumnos, individualmente, obtienen diferentes interpretaciones de las mismas experiencias construyendo y reconstruyendo sus conocimientos según saberes, esquemas y vivencias personales". (Chiarella, 2015 p. 705).

El maestro debe enseñar a los alumnos que es posible aprender por sí mismos, gracias a la experimentación, exploración, investigación y dejar que el alumno oriente su propio aprendizaje. De acuerdo con Ranière: "Se puede enseñar lo que se ignora si se emancipa al alumno, es decir, si se le obliga a usar su propia inteligencia". "No existe hombre alguno sobre la tierra que no haya aprendido alguna cosa por sí mismo y sin maestro explicador". (Ranière, 2004, p. 12,13).

La propuesta pretende desarrollar, por medio de la interacción entre los alumnos y profesores, habilidades y actitudes para el aprendizaje, promoviendo actividades de descubrimiento, análisis, investigación y procesamiento de la información. Estas actividades buscarán desarrollar la empatía docente, como recurso en la transmisión y motivación del aprendizaje significativo.

\section{La Empatía Docente}

El alcanzar un aprendizaje significativo muchas veces depende de la relación que el alumno establece con el profesor, que se ve reflejado en la empatía. El alumno suele escoger al profesor con el que se siente mejor y con más confianza para aprender un determinado curso. Desde la perspectiva empática, se crea una relación positiva entre profesor - estudiante, los alumnos tienden a interesarse más por las materias que son impartidas por profesores con los cuales tienen mayor cercanía. La labor de un profesor no 
debe limitarse exclusivamente a enseñar y evaluar el aprendizaje, es necesario transmitir una voluntad y actitud positiva respecto al curso.

La empatía desde el punto de vista psicológico, es ponerse en el lugar del otro para poder comprenderlo. Es la capacidad que lleva a entender las reacciones del estudiante, a ser conscientes de sus necesidades durante el proceso de formación. La relación entre el alumno y el profesor puede ser decisiva para mejorar su desempeño en el aula, pudiendo sin embargo, desarrollar empatía o rechazo.

De acuerdo con Uría: "La empatía o acción de sentir con el otro es propia de personas maduras, dentro del respeto y comprensión mutuos, captados por ambos. Puede ir lográndose en el aula y en este sentido o tiene por qué haber ausencia de actuación docente. El rechazo debe ser objeto de censura por parte del profesor, quien ha de propiciar en su lugar climas de empatía y encuentro que faciliten la coexistencia positiva del grupo." (Uría, 2001, p.170).

\section{Aprendizaje colaborativo}

A raíz de la evolución de los medios y la aparición de nuevos dispositivos y aplicaciones, las personas mantienen una mejor comunicación y conocimiento de otros entornos. Es común ver a los alumnos conversar a través del chat y compartir experiencias en las redes sociales, así se encuentren en el mismo ambiente físico. En lo que respecta a las actividades académicas, suelen recurrir a plataformas 0 espacios virtuales para facilitar la coordinación entre el grupo de estudio, y buscar respuestas rápidas en internet. Aunque a veces durante el desarrollo de un ejercicio de forma grupal la distribución de las tareas no es equitativa, son muchas las ventajas que puede ofrecer la interacción social en beneficio del aprendizaje.

Esta idea tiene origen en la teoría de Vygotsky, que manifiesta que el desarrollo cognitivo ocurre en la "zona de desarrollo próximo" (ZDP): nivel de conocimiento alcanzado en función de la interacción social, el comportamiento y el contexto. Esta habilidad puede ser desarrollada con la cooperación de un adulto o compañeros, y es más de lo que puede lograrse de forma individual.

"Vygotsky utiliza a menudo el término aprendizaje cooperativo en su discusión acerca de la evaluación de la zona de desarrollo próximo. El término no debe entenderse como un esfuerzo conjunto y coordinado para avanzar, en la que el socio más experto siempre está proporcionando apoyo en los momentos en que las funciones de maduración son inadecuadas. Más bien parece que este término se utiliza para referirse a cualquier situación en la que un niño está siendo ofrecido algún tipo de interacción con otra persona que está relacionado con un problema a resolver" (Kozulin, Gindis, Ageyew y Miller, 2003, p.54)

La interacción entre los miembros del grupo, permiten la realización de actividades de colaboración, las que mejoran con el uso de la tecnología, ante la aparición de las redes sociales, el uso de la nube y los espacios virtuales de trabajo colectivo. "El aprendizaje colaborativo, en una primera lectura, tiene una doble dimensión: colaborar para aprender y aprender a colaborar" (Rodríguez, 2001, p.64). Entre algunos de los efectos positivos del aprendizaje colaborativo, están: la motivación, la responsabilidad, la participación, el autoaprendizaje y el apoyo mutuo.

"El aprendizaje colaborativo mediado por entornos virtuales, debe centrarse en generar entornos de interacción para posibilitar los intercambios personales y la negociación de conocimientos nuevos. Las investigaciones en este campo adoptan el término CSCL, del inglés Computer Supported Collaborative Learning, y comparten el interés común por aprender cómo las tecnologías de la información y la comunicación pueden facilitar los procesos colaborativos en situaciones de enseñanza y aprendizaje, mejorando la interacción y el trabajo en grupo, así como los resultados de aprendizaje de los participantes, como base de conocimiento para elaborar propuestas pedagógicas desde esta perspectiva." Hernandez y Olmos (2011)

Para muchos jóvenes estudiantes, el trabajar en forma colaborativa en espacios digitales, es una característica natural y útil. Según Mugahed, Sharizan y Mi Yusuf: "Tanto los investigadores como los estudiantes tienen la oportunidad de experimentar en un aprendizaje colaborativo apoyado por computadoras y utilizar la plataforma de redes sociales." (Mugahed, et al, 2015. p. 1134).

En este entorno, es conveniente diferenciar dos términos que a veces son confundidos: el aprendizaje cooperativo y el colaborativo. El aprendizaje cooperativo o colectivo, permite al alumno un aprendizaje compartido, que favorece el reparto de actividades y de trabajo en grupo o en pares. De acuerdo con Fonseca y Redondo (2015, p. 55) "El reto para la accesibilidad, la buena educación y el trabajo colectivo beneficia el desarrollo de soluciones innovadoras". Esto puede favorecer a los estudiantes que tienen alguna dificultad y enriquecer a los más avanzados. Un aprendizaje colaborativo en cambio, permite que los alumnos utilicen una variedad de recursos tecnológicos para apoyar el desarrollo de actividades, transferencia de conocimiento y búsqueda de la información. Lo ideal es que el alumno pueda ejercitar habilidades de trabajo cooperativo y colaborativo en favor de un aprendizaje significativo.

\section{Aprendizaje significativo}

Durante el proceso de aprendizaje, es importante que el alumno pueda adquirir conocimientos de carácter significativo. Éste se produce cuando el alumno entiende lo que está aprendiendo, alcanzando un nivel de conocimiento y comprensión que se mantiene a largo plazo. Según Torre: "El aprendizaje significativo y sus elementos implicados (supra y subordinación de conceptos, inclusión comparativa y derivativa) posibilitan una comprensión progresiva de los conceptos, comprensión imprescindible para una adecuada utilización de estrategias." (Torre, 2002, p. 40).

Según Díaz (2002, p.19), el aprendizaje significativo es el que: "produce un cambio cognitivo, se pasa de una situación de no saber a saber. Es permanente: el aprendizaje que 
adquirimos es a largo plazo. Está basado en experiencia, depende de los conocimientos previos."

Desde el punto de vista de Ballester, "conseguir que el alumnado tenga estructuras de conocimiento potentes $\mathrm{y}$ significativas hace que se sienta bien y que mejore su autoestima, que se sienta interesado por lo que aprende y que le guste lo que hace; tiene un fuerte estímulo intelectual porque ve el resultado positivo de su proceso de aprendizaje, mantiene alta la moral del grupo y aprende a aprender" (Ballester, 2002 p. 18).

Es propósito del presente estudio, establecer estrategias metodológicas de trabajo colaborativo con actividades que mejoren la empatía docente; a través de la interacción producida por esta práctica, se busca desarrollar habilidades de autoaprendizaje, coevaluación y autoevaluación.

\section{Propuesta de enseñanza - aprendizaje}

La presente propuesta de enseñanza - aprendizaje se planteó en base a la siguiente hipótesis: "el trabajo colaborativo con actividades que fomenten la empatía docente, permite a los alumnos el mejoramiento de un aprendizaje significativo".

Para la comprobación de la hipótesis, se analizaron dos grupos de alumnos pertenecientes a semestres intermedios de la Facultad de Arquitectura y Urbanismo, uno experimental y otro de control. En el grupo experimental, se aplicaron prácticas que facilitaron las actividades de colaboración, durante las cuales el docente realizó un seguimiento grupal e individual, verificando el rendimiento de cada alumno.

Los profesores impartieron los mismos conceptos teóricos en ambos grupos, sin embargo los ejercicios para los alumnos del grupo experimental permitieron interactuar y debatir entre ellos, mediante un asesoramiento docente de forma permanente. Con actividades mediadas por la red, el docente tuvo la oportunidad de aplicar las acciones correctivas ante los posibles problemas, intentando mantener el nivel de desempeño homogéneo en el grupo.

Durante el proceso, se realizaron encuestas a estudiantes y profesores, las que sirvieron para comprobar cualitativamente la aceptación ante el experimento. El resultado de las evaluaciones se comparó finalmente entre los grupos de forma cuantitativa. Cabe resaltar que durante la realización de las encuestas y entrevistas, los alumnos mostraron mayor interés por el proyecto ejecutado y una constante comunicación con el profesor.

\section{Estrategias metodológicas}

Las actividades de práctica se orientaron principalmente al trabajo en equipo y de monitoreo docente, para comprobar el logro de los objetivos de aprendizaje. Se buscó la generación de tiempos y espacios para que los alumnos puedan compartir información y apoyarse mutuamente.
Para el desarrollo de la propuesta, se consideraron 5 estrategias de aprendizaje:

- Trabajo en equipo: los alumnos trabajaron de manera grupal con el fin de dialogar, evaluar, compartir información y recursos. Todo material fue colocado a disposición de todos los integrantes del grupo, se dispuso de un momento para discutir en aula y compartir los resultados de cada trabajo asignado.

- Estudio de casos: los alumnos debieron desarrollar un tema o proyecto específico en un determinado entorno geográfico. El problema fue establecido por el profesor y discutido en clase por cada grupo.

- Tutoría entre pares: los alumnos fueron co-responsables del trabajo de sus compañeros. En determinado momento del trabajo práctico, se formaron parejas para realizar una auto - evaluación y la apreciación desde el punto de vista del compañero. Este trabajo generó momentos de apoyo mutuo y de refuerzo de contenidos.

- Búsqueda de referentes web: el docente fomentó la investigación por medio de la búsqueda de información en bibliotecas virtuales y páginas web confiables. Los alumnos compartieron los contenidos en foros, aportando y evaluando los contenidos a utilizar.

- Monitoreo docente: el profesor realizó un seguimiento y control del avance del trabajo de los alumnos. Esta actividad permitió medir la asimilación del aprendizaje y realizar oportunamente acciones correctivas 0 de refuerzo de los temas tratados.

El docente realizó un monitoreo de los trabajos, controlando el avance de manera individual y grupal, con especial interés en las necesidades de los alumnos. "Las acciones de monitoreo / evaluación se llevan a cabo de forma permanente durante el desarrollo del proyecto y durante el proceso de adopción del modelo para incorporar las tecnologías de la información y comunicaciones." (Zea et al, 2003. p.9)

\section{Recomendaciones}

Los jóvenes tienen gran habilidad para relacionarse, estudiar o trabajar en equipo, por lo que el ambiente del aula se mantuvo en un ambiente de cordialidad, amistad y apoyo mutuo. Algunos profesores, tal vez por la diferencia generacional, suelen tener una limitada comunicación con sus alumnos, y esta brecha ser un impedimento para lograr un aprendizaje efectivo.

Con esta metodología, esta brecha de comunicación puede ser superada, el trabajo colaborativo y el monitoreo constante ayudan a una mejor empatía docente.

Para la efectividad del método, se recomienda la realización de las siguientes actividades: 
- Promover el trabajo grupal, el número de alumnos por grupo dependerá de la magnitud del proyecto y de los objetivos planteados en el silabo.

- Plantear un tema de trabajo que sea atractivo a los estudiantes, novedoso y actualizado. Los alumnos podrán desarrollar su creatividad y aplicar aspectos más avanzados y de especialidades, de acuerdo a su nivel en la carrera.

- La complejidad y los tiempos de ejecución de cada etapa del proyecto, dependerán de las capacidades planteadas para cada una.

- Solicitar las opiniones de los alumnos en relación con las actividades propuestas. Ellos deben ser partícipes de los acuerdos del aula, realizar diálogos y votaciones al respecto.

- Sugerir al grupo incluir algunas características adicionales que puedan mejorar la propuesta, como por ejemplo: ubicación, dimensiones del terreno, dimensiones mínimas y máximas de las áreas, posibles cambios de zonificación, topografía, etc.

- Brindar material didáctico en forma digital (videos, separatas, tutoriales, etc.) y sugerir el uso de tutoriales adicionales para resolver problemas fuera de clases.

- Motivar la comunicación constante y el intercambio de la información entre los integrantes del grupo.

- Utilizar las plataformas educativas para compartir material didáctico, en el caso de estudio, se empleó la plataforma Moodle.

- Los alumnos crearon grupos cerrados en redes sociales para la rápida comunicación entre el grupo.

- Realizar el monitoreo constante a fin de identificar a tiempo algún problema y poder hacer el refuerzo de los contenidos.

- Mantener la competencia entre los grupos de trabajo, para motivar la superación y apoyo de todos sus integrantes.

- Evaluar de forma periódica de los trabajos, de manera que el docente pueda llevar el control del desempeño de manera conjunta.

- Realizar entre los alumnos, una autoevaluación y coevaluación entre pares y dentro de los equipos.

\section{Resultados}

Con la aplicación de la presente propuesta en el grupo experimental, se transformó el aula de clase en un espacio de debate e interpretación de ideas colectivas, mediante el apoyo mutuo. Se logró fomentar la empatía docente, permitiendo el mejoramiento de un aprendizaje significativo, comprobando la hipótesis inicial planteada.

"Los docentes buscan crear ambientes física y socialmente óptimos para desarrollar sus actividades, ambientes que permitan dedicar más tiempo al aprendizaje, que integren más a los estudiantes". (Arias, 2005, p.12). Según Pujolás: "Se ha demostrado que cuando un alumno interactúa con otro para explicarle lo que ha aprendido se ve obligado a organizar sus ideas y se da cuenta, de sus errores y lagunas" (Pujolás, 2008. p. 13).

Referente a las deserción y el porcentaje de desaprobados, en el grupo experimental se consiguió el 100\% de alumnos aprobados, mayor asistencia y la reducción de tardanzas a las clases. A diferencia del grupo de control, que obtuvo un $15 \%$ de desaprobados y una deserción del $10 \%$.

Cuantitativamente, las evaluaciones obtenidas por el grupo experimental fueron mayores que los alumnos del grupo de control. El estudiante demostró mayor interés por el tema del curso, mayor disposición por aprender y cooperar con sus compañeros. Cada grupo aplicó sus habilidades de autoaprendizaje y apoyo mutuo, lo que sirvió en la solución de cualquier problema sin apoyo docente.

\section{Conclusiones}

Los resultados del proyecto experimental comprobaron la hipótesis que el trabajo colaborativo mejora la empatía docente y la adquisición de un aprendizaje significativo. Durante la aplicación de la propuesta, lo alumnos demostraron actitudes de responsabilidad, interés por aprender y mayor disposición al aprendizaje.

El aula de clases se convirtió en un lugar para compartir conocimiento dentro de una atmósfera amigable, los alumnos colaboraron con otros compañeros y mostraron mejor actitud en el desarrollo de sus trabajos. El profesor desarrolló una característica empática, mejorando la comunicación con sus alumnos y resolviendo a tiempo los problemas relacionados con el aprendizaje.

Es oportuno mencionar el esfuerzo que realizan los profesores en promover el aprendizaje mediante el trabajo colaborativo y el desarrollo de la empatía como estrategia de aprendizaje. Los profesores consiguen mejores resultados al ponerse en lugar de sus alumnos y reconocer sus dificultades. Las aulas podrán convertirse en ambientes generadores de saberes, donde los estudiantes son los promotores de su propio aprendizaje.

Se espera que el presente trabajo permita la realización de nuevas investigaciones y a los profesores mejorar las técnicas de enseñanza - aprendizaje con el uso de la tecnología. El éxito de una buena metodología, dependerá de la habilidad del profesor y de la búsqueda constante de nuevas estrategias docentes. 
SIGraDi 2016, XX Congress of the Iberoamerican Society of Digital Graphics 9-11, November, 2016 - Buenos Aires, Argentina

\section{Agradecimientos}

Es propicio agradecer a todos los alumnos que formaron parte de los grupos de trabajo y a la Facultad de Arquitectura y Urbanismo de la Universidad Ricardo Palma por su apoyo en la realización de la presente investigación.

\section{Referencias}

Aparici, R. (2013). Conectados en el ciberespacio. Universidad Nacional de Educación a Distancia. Madrid. UNED publicaciones.

Arias, J., Cárdenas, C., Estupiñán F. (2005). Aprendizaje Cooperativo. Universidad Pedagógica Nacional. Editora Guadalupe. Bogotá. Colombia.

Ballester, A. (2002). El aprendizaje significativo en la práctica. Cómo hacer el aprendizaje significativo en el aula. España. Recuperado de:

http://www.aprendizajesignificativo.es/mats/El_aprendizaje_signif icativo_en_la_practica.pdf

Cacheiro, M. (2014). Educación y Tecnología: Estrategias didácticas para la integración de las TIC. Uned Publicaciones. Madrid. España

Chiarella, M., Martín-Pastor, A. (2015). Pensamiento Gráfico y Diseño Colaborativo. Geometrías desarrolladas en composiciones plegadas arquitectónicas. Congreso Sigradi 2015. Project Information for Interaction. Florianópolis. Brasil.

Díaz, F. (2002). Estrategias docentes para un aprendizaje significativo. Una interpretación constructivista. Mc. Graw Hill.

Fonseca, D. Redondo, E (2015). Handbook of Reseach on Applied E-Learning in Engineering and Architecture Education. USA. IGI Global.

Hernández, A., Olmos, S. (2011). Metodologías de aprendizaje colaborativo a través de las tecnologías. Ediciones Universidad de Salamanca. España.

Kozulin, A., Gindis, B., Ageyew, V., Miller S. (2003) "Vygotsky's Educational theory in Cultural Context". The Zone of Proximal Development in Vygotsky's Analysis of Learning and Instruction. By Chaiklin, S. Cambridge University press. USA.

Mugahed, W. Sharizan, M and Mi Yusuf, L. (2015). "Effect of Engagement and Collaborative Learning on Satisfaction through the use of Social Media on Malaysian Higher Education" Research of Applied Sciences, Engineering and Technology. Maxwell Scientific Organization. Malaysia. Recuperado de:

https://www.researchgate.net/publication/281676330 Effect of e ngagement_and_collaborative_learning_on_satisfaction_through the_use_of_social_media_on_Malaysian_higher_education p. 1134
Niqui, C. (2011). La comunicación es vida. Reflexiones eclécticas sobre TICS y contenidos audiovisuales. Editorial UOC. Barcelona.

Universidad Politécnica de Madrid. (2008) Aprendizaje Cooperativo. Guías rápidas sobre nuevas metodologías. Recuperado de:

http://innovacioneducativa.upm.es/guias/Aprendizaje_coop.pdf

Pere, M. (2008). 9 Ideas Clave. El aprendizaje cooperativo. Editorial GRAÓ. Barcelona.

Pink, D. (2011). La Sorprendente verdad sobre qué nos motiva. Grupo Planeta. Spain.

Pozo, J. (2006). Teorías cognitivas del aprendizaje. Ediciones Morata. Madrid. España.

Pujolás P. (2008). 9 ideas clave. El aprendizaje cooperativo. Editorial Graó. Barcelona. España.

Ranière, J. (2003). El maestro ignorante. Cinco lecciones sobre la emancipación intelectual. Laertes, S.A. de ediciones. Barcelona, España. Recuperado de:

http://www.fundacion.uocra.org/documentos/recursos/articulos/El -Maestro-Ignorante-Ranciere.pdf

Rodríguez, J. (2001). Aprendizaje colaborativo en entornos virtuales. Anuario de Psicología. Vol. 32, N², Universidad de Barcelona. Recuperado de:

http://www.raco.cat/index.php/AnuarioPsicologia/article/viewFile/ $61669 / 88436$.

Rosetree, R. (2009). El poder de la empatía. Editorial Sirio. España.

Ruiz-Velasco E. (2013). Tecnologías de la información y la comunicación para la innovación educativa. Estudios. Posgrado en Pedagogía. Ediciones Díaz de Santos. UNAM. México.

Sagrá, A. González, M. (2004). La transformación de las universidades a través de las TIC: discursos y prácticas. Colección Educación y Sociedad Red. Editorial UOC. España.

Torre J. C. (2002). Aprender a pensar y pensar para aprender. Estrategias de aprendizaje. Narcea, S. A. de ediciones. Madrid. España.

Uría, Ma. E. (2001). Estrategias Didáctico-organizativas para mejorar los centros educativos. Narcea, S. A. Ediciones Madrid. España.

Zea, C., Atuesta, M., González, M. Montoya, J. y Urrego, I. (2003) Collaborative Learning Environments. A Response to the New Challenges of Colombian Education. Interactive Educational Multimedia, number 6. Colombia. Recuperado de:

http://www.conexiones.eafit.edu.co 